Скопје, Македонија

\title{
AGE FACTOR IN A MATH CONTEST PAPER FOR 5TH AND 6TH GRADE STUDENTS
}

\author{
BORISLAV LAZAROV AND ALBENA VASSILEVA
}

\begin{abstract}
The format of the Chernorizec Hrabar Math Tournament includes 5 divisions for students of two consecutive grades, e.g. 5th and 6 th grade students. A natural question is how appropriate is the common competition paper for any of two age strata. To clarify this issue we introduce the notion of age-factor index as the difference of the test-item difficulties calculated separately for any age stratum, e.g. $\Delta^{k}=T_{5}^{k}-T_{6}^{k}$ where $T_{5}^{k}$ and $T_{6}^{k}$ stand for the test-item difficulty of the $\mathrm{k}$-th test item for the 5th and 6th grade students' variation row respectively. The average value of $\Delta^{k}$ for the contest paper could be considered as a relative measure of reliability and uniformity of the contest paper for a particular target group. The study presents details about 2015 Chernorizec Hrabar contest paper for 5th and 6th graders and the application of the new concept in didactical analysis.
\end{abstract}

\section{INTRODUCTION}

The first issue of Chernorizec Hrabar math tournament $(\mathrm{ChH})$ was held in 1992 and there was just one competition paper for all participants who were advanced 9 th-11th grade students [2]. Since then the competition format changed several times and today's version includes 5 divisions for students of two consecutive grades, starting with 3 rd\&4th and finishing with 11th\&12th graders. The question about how appropriate is such format is natural. The possible answer strongly depends on the educational goal which differs for any of the age groups. However, some a posteriori calculated statistic parameters could help in didactical analysis of the competition paper.

\section{PARAmeters AND General Statistics}

The competition paper under consideration is the one for 5th\&6th graders, 2015 [4]. There were 20 multiple choice questions with one correct answer and 4 distractors each. The correct response is worth 7 points, the blank is

2000 Mathematics Subject Classification. 97C30.

Key words and phrases. age-factor index, multiple-choice competitions, Chernorizec Hrabar Math Tournament, high order thinking. 
3 and a wrong response is 0 . Such scoring provides guessing mathematical expectation of 28 points which guaranties rather poor result (take into account that a blank responses sheet is worth 60 points). Hence the students are not encouraged to guess the answer and we can accept that the correct answer was actually obtained by solving the problem. At least this is our assumption for the upper part of the variation row.

The total number of participants in this age group in 2015 was 1845 . The range of scoring was 14-140 points. The shape of the variation row (VR) is normal-distribution-like for the entire population (Figure 1).

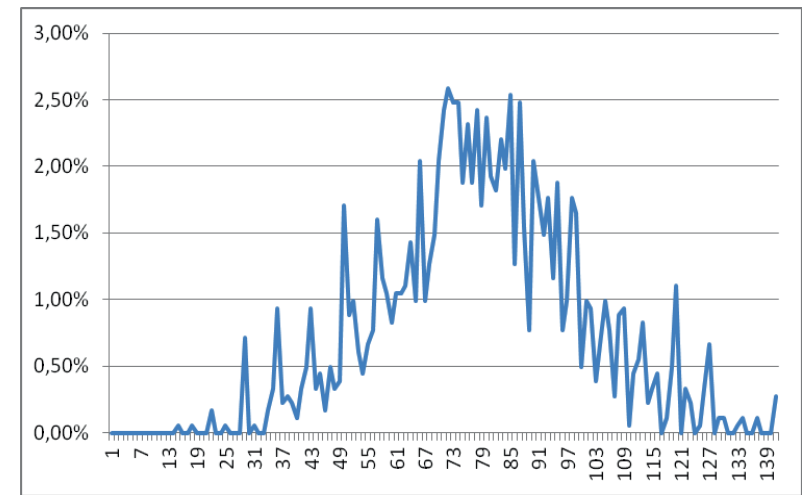

Figure 1. VR for the 5-6 graders, $2015 \mathrm{ChH}$ issue.

The population considered in our study is restricted to the participants in one particular competition center which is typical for middle-size town in Bulgaria. The 5th grade students were 56 and the 6 th graders were 54 .

The shift of the variation row of 6 th graders toward the one of the 5 th graders reflects a difference in the students' performance possibly caused by age (Figure 2). The 5th graders' graph is initially above the graph of the 6th graders. Somewhere between 70 and 80 points both graphs go together and then their position changes. Further we will study this quite natural phenomenon from didactic perspective with the help of a new statistical variable.

\section{The AGE-FACTOR INDEX}

The statistical method we apply is the standard one for a multiple-choice competitions paper, but we changed slightly the formula for calculating the test-item characteristics. Further we denote by $T_{n}^{k}$ the difficulty of the $k$-th test item $(k=1,2, \ldots, 20)$ for the $n$-th grade stratum $(n=5,6)$, and the discriminatory power by $D_{n}^{k}$ respectively. The values for $T_{n}^{k}$ are calculated as (100 minus the percentage of the number of correct responses), i.e. as the percentage of the incorrect and the blank responses altogether. The values 


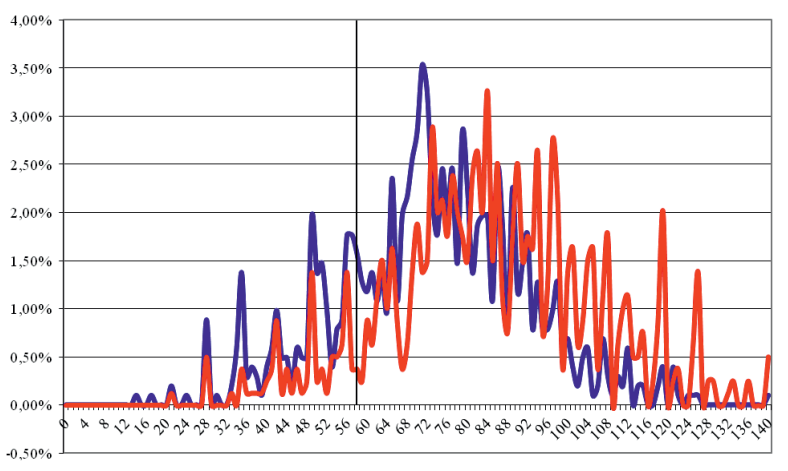

FiguRE 2. VRs for the 5 and 6 graders (blue line and red line, respectively).

for $D_{n}^{k}$ are half of the difference between the difficulties for the fourth and the first quartile.

We define the age-factor index (AFI) of the $k$-th test item as the difference $\Delta^{k}=T_{5}^{k}-T_{6}^{k}$. Let us note that the variable $\Delta^{k}$ is not only age-dependent. However, we will use it in our didactic analysis as an additional instrument that indicates mainly age-dependent features of a particular test item. The distribution of $\Delta^{k}$ for the competition paper under consideration is given in Figure 3.

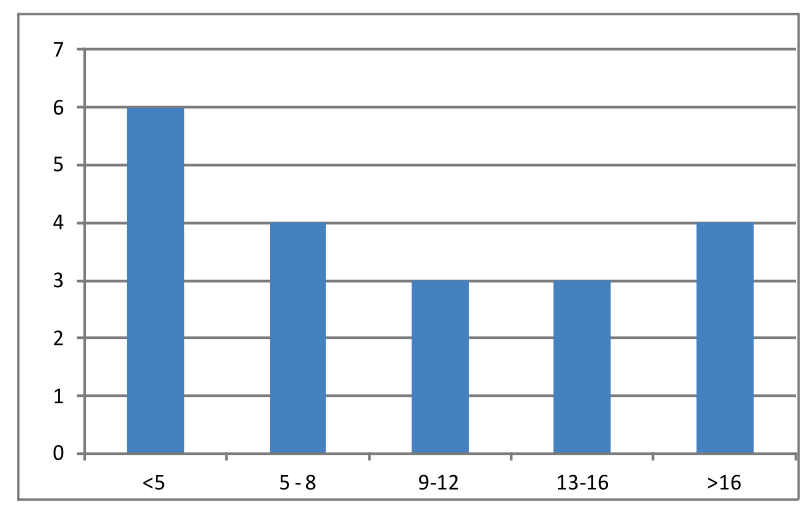

FIGURE 3. Distribution of $\Delta^{k}$.

We are also interested in the average value $\Delta$ of AFIs for the entire competition paper. It could be interpreted as the magnitude of the shift of the graph of the 6th graders' VR with respect to the 5th graders' one.

Our recent statistics clearly shows that in some small groups the age factor does not play significant role in student performance, especially for senior secondary school. For instance, the 11th graders of a particular 
competition center showed in average better results than 12th graders in the 2015 issue of $\mathrm{ChH}$. However, in the middle school $\Delta$ occurs to be close to 10 for the last two consecutive issues of $\mathrm{ChH}$ and we think this could be not just a random coincidence.

\section{Didactic analysis}

In our previous study [5] we detected that the AFI of some problems which concern extracurricular topics is less than the AFI of test items closely related to the school curriculum. Our conjecture was that the math knowledge and skills formed in extracurricular activities are more sustainable than the ones built in the frame of school curriculum. The next three examples will show another side of age dependent math abilities.

TI 3. Let us define two new operations for the numbers $a$ and $b$ :

$$
a \natural b=a b-a-b \text { and } a \sharp b=a b+a+b .
$$

For example, $5 \llbracket 2=5 \cdot 2-5-2=3,5 \sharp 2=5 \cdot 2+5+2=17$. Which is the number $x$ in the diagram?

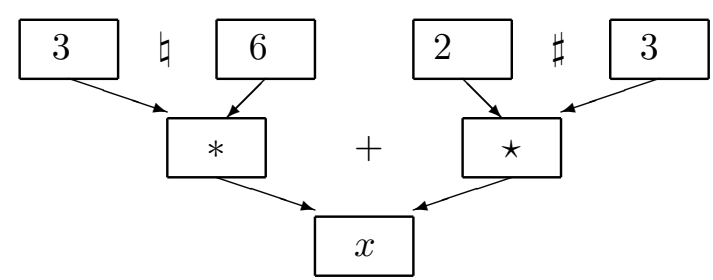

$\begin{array}{lllll}\text { (A) } 20 & \text { (B) } 12 & \text { (C) } 22 & \text { (D) } 18 & \text { (E) none of these }\end{array}$

Solution. $3 \natural 6=9,2 \sharp 3=11, x=9+11$.

Correct answer (A).

Comment. The test-item statistics is: $T_{5}^{3}=32.1 ; D_{5}^{3}=21.4 ; T_{6}^{3}=$ $11.1 ; D_{6}^{3}=7.1$ and $\Delta^{3}=21.0$. The introduction of a new concept in a contest paper is always risky. This test-item combines the very well known technique of calculating expression value in a diagram with the also familiar funny-looking operations. Our previous research claims that the easiest problems about arithmetic expressions are of diagram type [1]. However, the large value of $\Delta^{3}$ indicates that such combination of ideas is inside the 6 th graders zone of proximal development (ZPD) but for the one third of the five graders it is beyond their ZPD [6].

TI 12. Alex, Bobby and Charlie thought of the same two-digit number. Alex multiplied the number by 3 , then added 3 to the result, then divided the new result to 3 , subtracted 3 from the quotient and obtained the number $A$. Bobby added 3 to the initial number, multiplied the result to 3 , subtracted 3 from the product, divided the result by 3 and obtained the number $B$. Charlie first subtracted 3 from the initial number, then multiplied the result by 3 , added 3 to the product, divided the sum to 3 and obtained the number $C$. How are ordered the numbers $A, B$ and $C$ ? 

(A) $\mathrm{A} \geq \mathrm{B} \geq \mathrm{C}$
(B) $\mathrm{C} \geq \mathrm{A} \geq \mathrm{B}$
(C) $\mathrm{A} \geq \mathrm{C} \geq \mathrm{B}$
(D) $\mathrm{B} \geq \mathrm{A} \geq \mathrm{C}$
(E) $\mathrm{C} \geq \mathrm{B} \geq \mathrm{A}$

Solution. Let $X$ be the initial number. Performing the operations, we obtain

$$
\begin{aligned}
& A=(X \cdot 3+3) / 3-3=X+1-3=X-2, \\
& B=((X+3) \cdot 3-3) / 3=(3 X+9-3) / 3=X+2, \\
& C=((X-3) \cdot 3+3) / 3=(3 X-9+3) / 3=X-2 .
\end{aligned}
$$

The problem may be solved by using a specific value of $X(X \geq 3$ for 5 th graders), e.g. if $X=12$, then $A=10, B=14, C=10$.

Correct answer (D).

Comment. This test-item statistics is: $T_{5}^{12}=51.8 ; D_{5}^{12}=28.6 ; T_{6}^{12}=$ 18.5; $D_{6}^{12}=33$ and $\Delta^{12}=21$ (this is the largest AFI in the competition paper). Problems of this type appear in $\mathrm{ChH}$ competitions papers as easy ones, but usually they are written in schematic format [3], e.g. the difficulty of the quite similar test-item 10 from the 2015 competition paper was 16.7 in the 5 th grade stratum. The verbal format of the operation description appears to be insurmountable for half of the 5th graders but it causes no troubles in 6th grade.

TI 15. How many natural numbers are there, whose sum of digits equals 7 and product of digits equals 8 ?
(A) 4
(B) 6
(C) 8
(D) 10
(E) 12

Solution. The digits of the desired numbers are factors of 8 . A digit 8 makes the sum of the digits greater than 7 . Thus only 1, 2, and 4 could be possible digits. There are two cases.

(1) 4 is among the digits; there cannot be another 4 , hence it must be exactly one 2 to get the product 8 ; to complete the sum exactly one 1 is needed; there are 6 numbers formed by the digits 4,2 and 1 .

(2) 4 is not among the digits; there must be 3 digits 2 to get the product 8; to complete the sum exactly one 1 is needed; there are 4 numbers formed by three 2 and one 1 .

Correct answer (D).

Comment. The test-item statistics is: $T_{5}^{15}=91.1 ; D_{5}^{15}=10.7 ; T_{6}^{15}=$ $63.0 ; D_{6}^{15}=28.6$ and $\Delta^{15}=28$. The problem is of complex type. The first step refers to divisibility and the second step is combinatorial. It is no surprise for us that the difficulty is high for both strata. The high value of AFI confirms the conjecture that combining math ideas is beyond ZPD of a considerable fraction of advanced students in 5th and 6th grade. However, the statistics gives reason to claim that for one third of the 6th graders such complex knowledge and skill could be achieved, i.e. complex problems are in their zone of actual development (ZAD) [6].

TI 6. The long-eared owl makes calls at equal intervals; the duration of a call is ignored. An owl needs 20 seconds to make 5 calls. How many seconds are needed for an owl to make 25 calls? 

(A) 108
(B) 100
(C) 125
(D) 96
(E) 120

Solution. There are 4 intervals between the five calls, i.e. each interval is $20 / 4=5$ seconds long. There will be 24 intervals between 25 calls, therefore $24 \cdot 5=120$ seconds will be needed to make 25 calls.

Correct answer (E).

Comment. The statistics is: $T_{5}^{6}=85.7 ; D_{5}^{6}=3.6 ; T_{6}^{6}=85.2 ; D_{6}^{6}=7.1$ and $\Delta^{6}=1$. To our surprise, the statistical difficulty is too high for this easy one-step problem. Moreover, every issue of $\mathrm{ChH}$ includes such kind of problems, even in the competition papers for 3-4 grades. The small $\Delta^{6}$ value says that it is an equally slippery problem for both age strata. On the other hand, the small $D_{5,6}^{6}$ means that the problem appears tricky for both advanced and less able students. The most frequent students response was (B) which corresponds to the "solution": 5 times more calls need 5 times more time. Maybe the accommodation of this test item in the beginning of the competition paper caused such misunderestimation ;-).

TI 10. Which is the arithmetic operation replaced by the symbol $\nabla$ in the equation

$$
(555+5-55) \nabla(5-5: 5)-5=2015 ?
$$
(A) addition
(B) subtraction
(C) division
(D) multiplication

(E) none of the operations is appropriate

Solution. $505 \nabla 4-5=2015 ; 505 \nabla 4=2020 \Longleftrightarrow 505 \cdot 4=2020$. Correct answer (D).

Comment. The statistics is: $T_{5}^{10}=7.1 ; D_{5}^{10}=3.6 ; T_{6}^{10}=5.6 ; D_{6}^{10}=10.7$ and $\Delta^{10}=2$. Here the AFI's value tells us that this problem was equally easy for both age strata.

TI 4. In the summer of 2016 we will celebrate the 100th anniversary of Dimitar Spisarevski. On December 20, 1943, lieutenant Spisarevski died while destroying an enemy bomber attacking Sofia. How old was the lieutenant when he accomplished this feat?
(A) 43
(B) 16
(C) 27
(D) 20
(E) 36

Solution. Dimitar Spisarevski was born on July 19, 1916, and therefore he was $43-16=27$ years old.

Correct answer $(\mathrm{C})$.

Comment. The statistics is: $T_{5}^{4}=5.4 ; D_{5}^{4}=7.1 ; T_{6}^{4}=7.4 ; D_{6}^{4}=10.7$ and $\Delta^{4}=-2$. The values of $D_{5,6}^{4}$ advocate that the problem provides better opportunities for the more prepared students. The negative AFI is quite unusual phenomenon but it happened. Our explanation is that this is another underestimated test item by some of the 6th graders.

Historical note. The birth date of Dimitar Spisarevski is not clarified. About 4000 civilians died during the American bombings of Sofia in 1943-1944. 


\section{Conclusions}

The VR of the entire population evidences that our competition paper provides enough space for the most prepared and gifted students to manifest their knowledge and skills. We accept that the average AFI value is normal to be close to 10 for the 5-6 graders. The distribution of $\Delta^{k}$ shows that half of the test items are equally suitable for 5 th and 6 th grade. The other half of the test items gives a little advantage to the elder stratum but also encourages the younger students to study mathematics deeper.

The examples from the previous section confirm our note that AFI alone cannot provide enough information about the quality of a test item. However, the relatively large AFI values focus our attention on some significant age dependent sides of student's cognitive development. The AFI values in the first three examples hint that the ability to combine sophisticated math ideas is a sort of thinking of very high order. The values of $D_{5,6}^{k}, k=3,12,15$, support the thesis that such ability appears to be in progress between 5 th and 6 th grade.

\section{COMPETING INTERESTS}

Authors have declared that no competing interests exist. Autors' contributions: Borislav Lazarov - competition paper, conceptual frame and research design; Albena Vassileva - data processing and statistics.

\section{ACKNOWLEDGMENTS}

This work has been done in the frame of Chernorizec Hrabar Educational and Research Program of the Institute of Mathematics and Informatics of the Bulgarian Academy of Sciences. Authors thank Irina Sharkova for capturing the source data for the statistics.

\section{REFERENCES}

[1] A. Vassileva, B. Lazarov, A study of the effect of formal and verbal formulation of test units in 3rd and 4th grade, in: Proceedings of International conference Synergetics and reflection in mathematics education, 10-12 September 2010, Bachinovo, Bulgaria, 344-350 (in Bulgarian)

[2] B. Lazarov, A New Tournament in Bulgaria, Mathematics Competitions, Vol 6, No 1, Canberra (1993) 47-55.

[3] B. Lazarov, Twenty third autumn tournament in mathematics and informatics Chernorizec Hrabar, IMI-BAS, Sofia (2015), 20-25. (in Bulgarian) ISBN 978-9548986-42-7

[4] B. Lazarov, Twenty forth autumn tournament in mathematics and informatics Chernorizec Hrabar, IMI-BAS, Sofia (2016), 20-25. (in Bulgarian) ISBN 978-9548986-44-1

[5] B. Lazarov, A. Vassileva, Age-dependent specifics of students performance on a same competition paper, in: Mathematics and education in mathematics, Proceedings of the Forty Fifth Spring Conference of the Union of Bulgarian Mathematicians, Pleven, April 6-10, 2016, 242-247. (in Bulgarian) 
[6] L. Vygotsky, Dynamics of cognitive development in relation to student learning. Mental development of children in the learning process, Moscow-Leningrad, (1935) 33-52. (in Russian).

Department of Education, Institute of Mathematics and Informatics SOFIA, Bulgaria.

E-mail address: lazarov@math.bas.bg

Department of Education, Institute of Mathematics and Informatics SOFIA, Bulgaria.

E-mail address: albena@math.bas.bg 